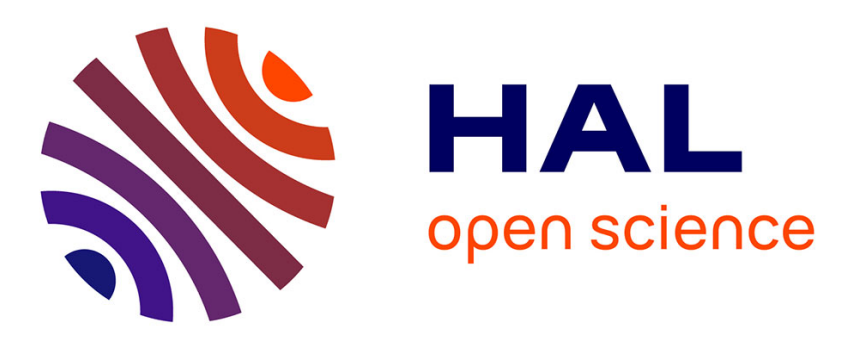

\title{
Manufacturing of 3D Helical Microswimmer by AFM Micromanipulation for Microfluidic Applications
}

Gilgueng Hwang, Christophe David, Alisier Paris, Dominique Decanini, Ayako Mizushima, Yoshio Mita

\section{- To cite this version:}

Gilgueng Hwang, Christophe David, Alisier Paris, Dominique Decanini, Ayako Mizushima, et al.. Manufacturing of 3D Helical Microswimmer by AFM Micromanipulation for Microfluidic Applications. IEEE Transactions on Semiconductor Manufacturing, 2021, 10.1109/TSM.2021.3082902 . hal03415804

\author{
HAL Id: hal-03415804 \\ https://hal.science/hal-03415804
}

Submitted on 5 Nov 2021

HAL is a multi-disciplinary open access archive for the deposit and dissemination of scientific research documents, whether they are published or not. The documents may come from teaching and research institutions in France or abroad, or from public or private research centers.
L'archive ouverte pluridisciplinaire HAL, est destinée au dépôt et à la diffusion de documents scientifiques de niveau recherche, publiés ou non, émanant des établissements d'enseignement et de recherche français ou étrangers, des laboratoires publics ou privés. 


\title{
Manufacturing of 3D Helical Microswimmer by AFM Micromanipulation for Microfluidic Applications
}

\author{
Gilgueng Hwang*, Christophe David, Alisier Paris, Dominique Decanini, Ayako Mizushima, and Yoshio Mita
}

\begin{abstract}
Manufacturing of microsystems based on complex three-dimensional microstructures requires critical steps such as mass calibrations and integration process. However, such steps involve several manipulation steps which are not well controlled thus the fragile 3D microstructures could be damaged or destroyed. In this work, we have demonstrated an AFM based direct mass measurement and manufacturing of 3D helical microswimmer. The proposed method shows to be rapid, repeatable and minimally-destructive thanks to the precise force control and manipulation by AFM. The proposed micromanipulation steps consist of the pick-measure-integrate manipulation steps using van der Waals force-based attachment and the mass measurement by resonant frequency shift. For the testing structures, we fabricated the 6 different types of 3D helical microswimmers vertically fabricated on the conical shape microneedle supports for uniform surface metallization and facile detachment. With the mass measurement sensitivity of $25 \mathrm{~Hz} / \mathrm{pg}$ and the direct integration to microfluidics, we successfully demonstrated the 3D propulsion and non-contact micromanipulation by 3D helical microswimmer in microfluidics.
\end{abstract}

Index Terms-Atomic force microscopy, Mass measurement, Microassembly, Microfluidics, Microswimmer

\section{INTRODUCTION}

$\mathrm{T}$ hree-dimensional micro/nanostructures could be a promising building block for a wide range of applications. Among different designs, 3D helical nanostructures have been fabricated from gold [1], silver [2], photonic crystals [3] for electromagnetic components or photonic metamaterials. They have drawn great interests in the photonics/optics [1,3], force/displacement sensors [4,5], wireless actuators [6,7]. These 3D helical microstructures are promising building blocks towards micro electromechanical system (MEMS) sensors or actuators. Concerning the fabrication methods, the self-scrolling technology by strain-engineering of epitaxially

A part of this work is supported by Japan Society for the Promotion of Science (JSPS) through invitational fellowships for research in Japan. The test microstructure design, 3D nanolithography and AFM measurements were supported by the French RENATECH network through its C2N technological facility. Metallic layer deposition by sputtering was supported by MEXT/VDEC Nanotechnology Platform in The University of Tokyo. ( ${ }^{*}$ Corresponding author: Gilgueng Hwang)

G. Hwang and Y. Mita are with the Laboratory for Integrated Micro Mechatronic Systems, French National Centre for Scientific Research, Institute of Industrial Science, The University of Tokyo, Tokyo, Japan.

G. Hwang, A. Mizushima, and Y. Mita are with the Department of Electrical Engineering and Information Systems, The University of Tokyo, Tokyo, Japan.

G. Hwang, C. David, A. Paris, and D. Decanini are with the Centre for Nanoscience and Nanotechnology, Paris-Saclay University, Palaiseau, France. (e-mail: gilgueng.hwang@c2n.upsaclay.fr, hwang@if.t.u-tokyo.ac.jp) grown semiconductor bilayers has been widely studied [4-7]. Other materials like metals or dielectrics could also form helical structures by glancing angle deposition method [8]. Recently a direct laser writing by two-photon absorbed polymerization method simplified the 3D microfabrication and also allowed more complex geometries [9]. Combining it with the electrolytic growth, metallic (gold) helical structures could be fabricated for photonic metamaterials [10]. Deposition by sputtering of metallic layers could fabricate the metal-polymer helical structures for the magnetic actuation [11]. This magnetically actuated 3D helical microswimmers have a great interest to serve as mobile micromanipulators [11,12], physical sensors in microfluidics $[13,14]$, neuroregenerative therapy [15], soft swimmers [16] or water remediation [17]. However, for such applications, precise mass measurements of such 3D helical microstructures are crucial to their physical force calibrations. Precise mass measurements of such 3D helical microswimmers are important mainly for two reasons. First, when the microswimmers are applied to the force sensors [13] or flow sensors [14], the precise force or flow sensing rely highly on the precise mass measurements of microswimmers to complete the propulsion matrix. Second, the enhancement of propulsion performance by optimal designs of microswimmers require precise propulsion force measurements of each designs which require the precise mass measurements of microswimmers. Concerning the capillary force effect, since the microswimmers are fully immersed with surrounding liquid medium and their surfaces are hydrophilic thus to minimize the capillary effect, the enhanced propulsion force by optimal designs allows them to overcome the capillary force and also the gravity to be able to swim out of the substrate. Considering the absence of an established mass measurement method for such 3D microstructures, an approach to combine the measurement and the calculation has been utilized [13].

In the case of the magnetically actuated 3D helical microswimmers fabricated by two-photon absorbed polymerization and additional active metallic layers coating, mass estimation requires the precise volume estimations of such 3D microstructures based on the lithographical voxel size estimations from electron microscope images, the resist density measurements and the metallization volume estimations. Additional process like metallization in the fabrication causes the volumetric variation in the final volume and mass from the initial design thus expected to optimize the process. This requires mass estimation of such composite 3D materials. Conventionally the mass was able to be estimated by the computational volume estimation of the $3 \mathrm{D}$ structures and 
TABLE I

MASS MEASUREMENT METHODS OF MiCROSTRUCTURES

\begin{tabular}{|c|c|c|c|}
\hline & MEMS Sensor & $\begin{array}{c}\text { In-situ SEM } \\
\text { nanomanipulation }\end{array}$ & $\begin{array}{c}\text { Proposed } \\
\text { Method }\end{array}$ \\
\hline Environment & Air & Vacuum & Air \\
\hline Measurement & Hysteresis/Drift & Drift & Controlled \\
\hline $\begin{array}{c}\text { Sample } \\
\text { Contamination }\end{array}$ & $\begin{array}{c}\text { Mass variations } \\
\text { (addition or } \\
\text { loss) }\end{array}$ & $\begin{array}{c}\text { Mass variations } \\
\text { (glue or ebeam } \\
\text { deposition) }\end{array}$ & Minimum \\
\hline $\begin{array}{c}\text { Sample } \\
\text { placement }\end{array}$ & Not-controlled & Controlled & Controlled \\
\hline $\begin{array}{c}\text { Sample } \\
\text { attachment }\end{array}$ & Van der Waals & $\begin{array}{c}\text { Chemical glue or } \\
\text { ebeam deposition }\end{array}$ & Van der Waals \\
\hline $\begin{array}{c}\text { Reconfigure/ } \\
\text { Repeatable }\end{array}$ & No & No & Yes \\
\hline $\begin{array}{c}\text { Process time } \\
\text { Long } \\
\text { (sample } \\
\text { transport) }\end{array}$ & $\begin{array}{c}\text { Long } \\
\text { (require vacuum, } \\
\text { deposition) }\end{array}$ & $\begin{array}{c}\text { Short } \\
\text { (direct } \\
\text { measurement) }\end{array}$ \\
\hline
\end{tabular}

deposited metallic layers [13]. The computational model estimation is limited to the simple models and could derive with errors from the real value. The Ref [13] describes a way for the mass estimation of single helical microswimmers with constant filament size by combining the computational model and the experiments. The estimation of mass was completed with the error estimations for the fabrication steps such as the 3D lithography and the metal layer deposition. However, applying the same method to more complex 3D helical microstructures with non-constant filament sizes as shown in this work could derive more important estimation errors. Therefore, a way of direct mass measurement should be explored. A major challenge of such experimental measurements of 3D composite microstructures is due to their fragile mechanics which can cause the loss of materials from damage during the measurement. Moreover, the measured structures need to be integrated to microfluidic chips for their propulsion tests and applications. Further propulsion characterizations in microfluidic environment should follow to be able to complete the propulsion force calibrations. This series of the estimation steps involves many sources of the estimation error especially in the volume estimations. This also requires an additional step of translocating manipulation of the structures which can easily damage the microstructures. Therefore, a way of direct mass measurement and microfluidic integration is crucial to establish.

Table I summarizes other conventional approaches. One of the most widely used method is to use MEMS based resonant mass sensors $[18,19]$. Considering the dependency of precise positioning of the structure to measure with the MEMS sensors, a precise micromanipulation process is essential. Coupling the resonant sensors and In-situ scanning electron microscope (SEM) nanomanipulation could be considered. However, measuring the mass under vacuum and electron-beam exposure, the process could be longer than in air and could also contain some artifacts [20].

Our previous work [21] described the AFM based direct mass measurement strategy for 3D helical microstructures. In this work, we further extended the mass calibration to the manufacturing of 3D helical microswimmers in microfluidics to demonstrate their microfluidic applications with 3D propulsions and non-contact micromanipulation. It should be

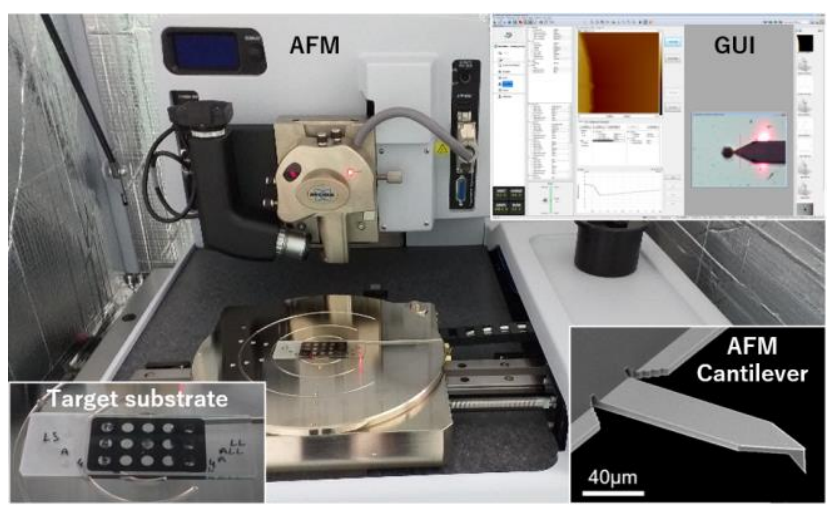

Fig. 1. AFM Micromanipulation system with a GUI for the control, imaging and sensing by AFM (inset photo, top-right), the AFM cantilever (bottom-right) and the target substrate (bottom-left).
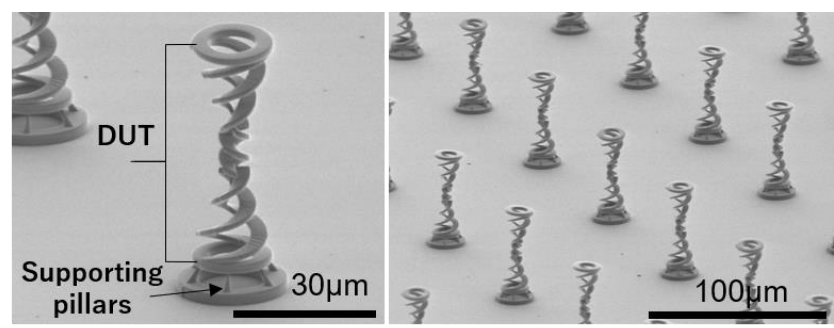

Fig. 2. SEM images of an array of the 3D helical test microstructures fabricated by two-photon absorption laser 3D nanolithography and metallization.

noted that the AFM based mass calibration and better controlled manufacturing process allowed to improve the quality of characterizations of the microfluidic propulsion and manipulation. The proposed method is able to perform precise manipulation with force control in air. This is a rapid, reliable and less-destructive method to measure the mass of such 3D microstructures thanks to the highly precise positioning accuracy with force sensing capability of AFM. The method is performed by AFM micromanipulation steps combining both the tapping mode and the contact mode manipulation. Using AFM as micro manipulator as well as mass sensor, the mass measurements can be directly done on the fabrication substrate containing the test samples. Compared to conventional approaches using MEMS mass sensors or in-situ SEM nanomanipulation, this measurement can avoid additional transportation steps from the fabrication substrate to the MEMS mass sensor (Table I). Since the proposed method uses only van der Waals force, the loss or addition of the mass could be avoided compared to other approaches based on chemical glues or deposition. Moreover, the reversible detachment/attachment feature of the proposed method allows repeatable measurements of the same or multiple samples with the same AFM tip. The paper describes first on the system overview of the AFM micromanipulation system and the fabrication of 3D helical microstructures fabricated on the supporting microneedle array. Then we describe the chemical-free micromanipulation strategy for the mass measurements with minimal addition or loss of the mass. Finally, the microswimmers integrated to microfluidics will demonstrate the 3D non-contact micromanipulation based on hydrodynamic 


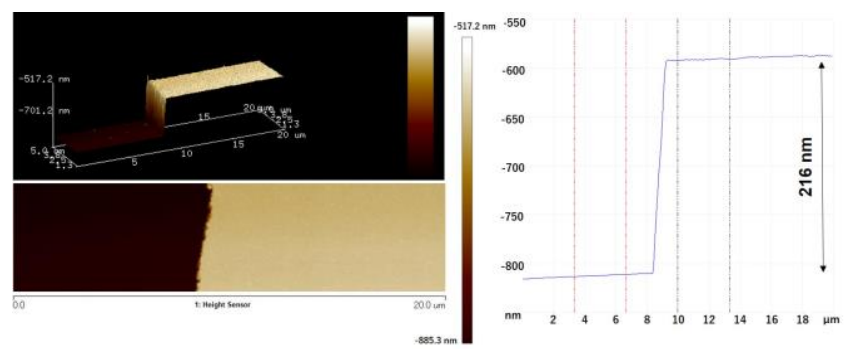

Fig. 3. Deposited metal layer (bright part in the left) thickness analysis with the topological profile (right) measured by AFM.

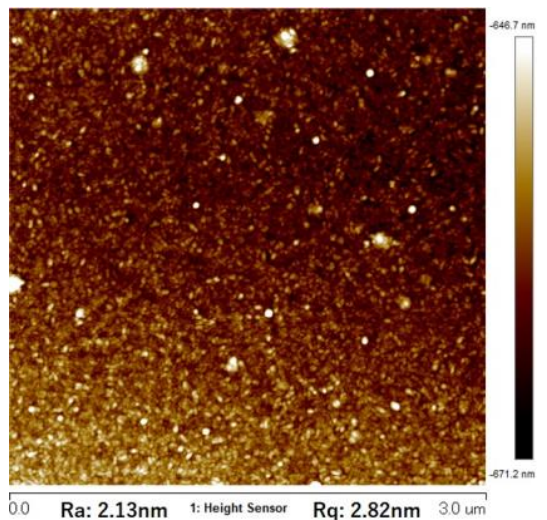

Fig. 4. Surface roughness analysis of the deposited metal layers by AFM imaging.

vortex generated from the motion of helical swimmers.

\section{SYSTEM OVERVIEW}

\section{A. Micromanipulation System with Atomic Force Microscope}

We used an AFM system (Bruker Icon) with a visible apex AFM tip (OTESPA-R3). The AFM tip was chosen to visualize the tip during the manipulation process to avoid unnecessary damage to the helical structures. The tetrahedral tip with the sharper tip (tip radius less than $10 \mathrm{~nm}$ ) was used for high lateral resolution. The motion of the cantilever is controlled by users through a graphical user interface (GUI) for the surface topography analysis, the precise force applications, the resonant frequency analysis with thermal tuning and manipulation of the structures. The nominal resonant frequency and the spring constant of the AFM cantilever were $300 \mathrm{kHz}$ and $26 \mathrm{~N} / \mathrm{m}$ respectively. The resonant frequency was calibrated by thermal tuning for each measurement. Prior to the manipulation, a substrate with the fabricated microstructures and a target substrate were placed on the sample stag e. The target substrate contains an array of chambers for the identifications of the placed microstructures after the mass measurement step. For the integration to microfluidic devices, a glass substrate was used as the target substrate and it is followed by an $\mathrm{O}_{2}$ plasmas bounding of the glass substrate containing the measured helical microstructure with a PDMS (Polydimethylsiloxane) fluidic channel layer prepared by mold casting process.

\section{B. Fabrication of $3 D$ Helical Microstructures}

We designed and fabricated the triple bi-helical test

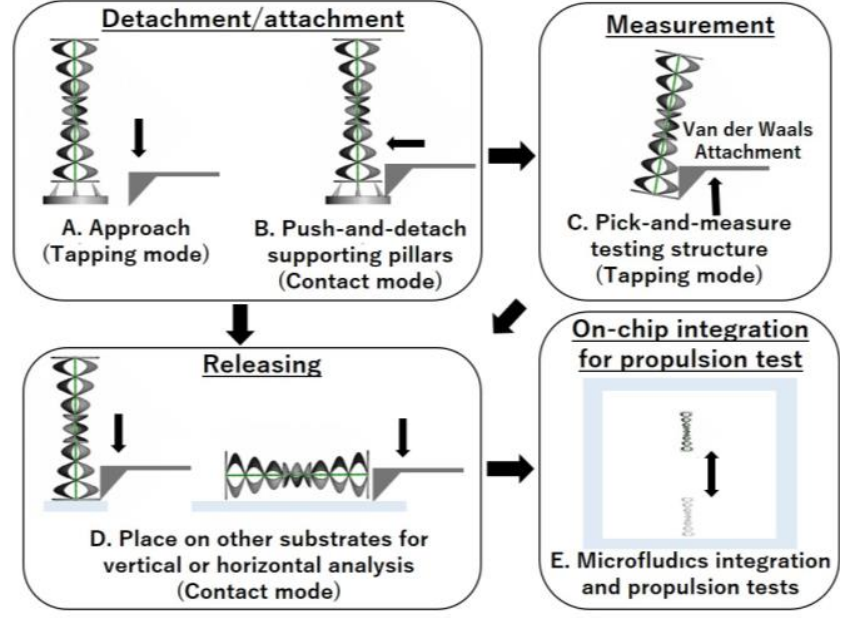

Fig. 5. Schematic concept of an AFM based pick-measure-integrate process of 3D helical microstructures for their microfluidic integrations for propulsion test. The process consists of the sequences 1 . detachment/attachment, 2. measurement, 3. releasing onto other substrate and 4. microfluidic integration for propulsion tests.

microstructures with polymer and metallic layers which can be applied for micromanipulation of microscale objects in microfluidic environments [12]. These 3D helical microstructures were demonstrated to be applied for non-contact micromanipulation based on hydrodynamic trapping by vortex. The helical microstructures were fabricated by two-photon polymerization using 3D laser nanolithography system (Nanoscribe $\mathrm{GmbH}$ ) and surface metallization of ferromagnetic metal layer for magnetic propulsion. Conventionally such helical microstructures were fabricated horizontal to the substrate for a better stability during fabrication process considering that such 3D microstructures could be damaged or detached by the surface tension at the wet/air interface during development and drying process. Although the horizontally fabricated microstructures have an advantage on the fabrication stability, there are two critical issues. First, the 3D microstructures cannot be uniformly coated with metallic layers due to a shadowing effect during the metallic layer deposition even by sputtering. This could decrease the performance of the device and the deposited materials could be lost due to weak bounding to surface. Second, the detachment process necessary for the mass measurement and the integration to microfluidics could be much more complex due to the increased number of the surface contact. Moreover, the surface contacts are through the whole body of the helical microstructures thus there are much higher risk of damaging the active part of helical structures during the detachment process. In these reasons, we proposed to design and fabricate the helical microstructures vertical to the substrate. The vertically standing helical microstructures were fabricated on top of the supporting structure with conical shape microneedle array which elevate and maintain the structures 5 $\mu \mathrm{m}$ above the substrate thus to avoid shadowing effect during deposition of other layers. The metallic layers $(\mathrm{Cr} / \mathrm{Ni}$ 10/200 $\mathrm{nm}$ ) were deposited by sputtering to maximize the deposition uniformity. The minimum contact at one side of ring between the conical shape microneedle array and the substrate allows 


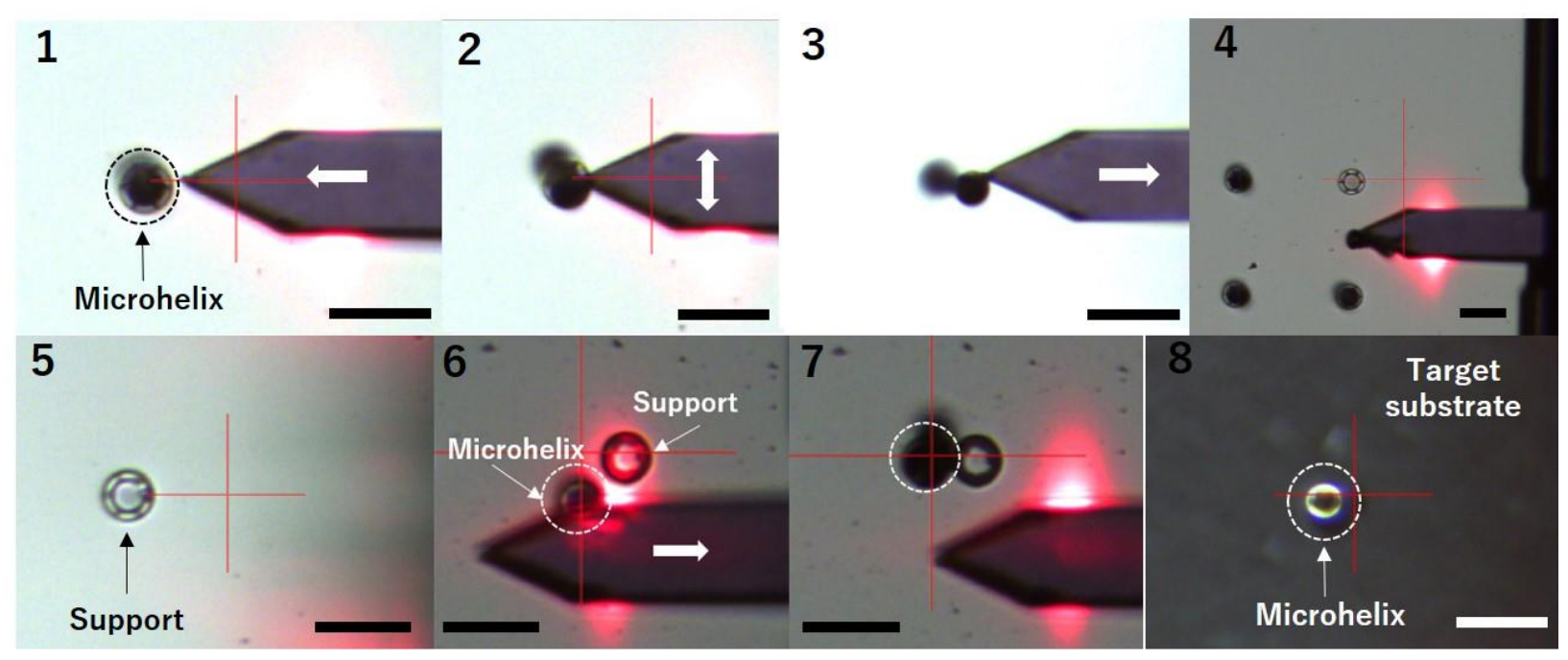

Fig. 6. Pick-measure-place micromanipulation by AFM: 1. AFM tip is placed on top of the bottom support ring between two supporting pillars and move towards the center of microhelix, 2. AFM tip is moved laterally to disconnect the microhelix from supporting pillars, 3. Retraction of AFM tip out of the microhelix, 4. Moving up the picked-up microhelix with AFM tip, 5. Verification of the remained bottom supporting ring, 6 . The pick-up microhelix is approaches back to the support, 7. The microhelix is placed back near the support, 8 . The microhelix is placed on other substrates for further analyses or for microfluidic integration. Scale bars are $40 \mu \mathrm{m}$.

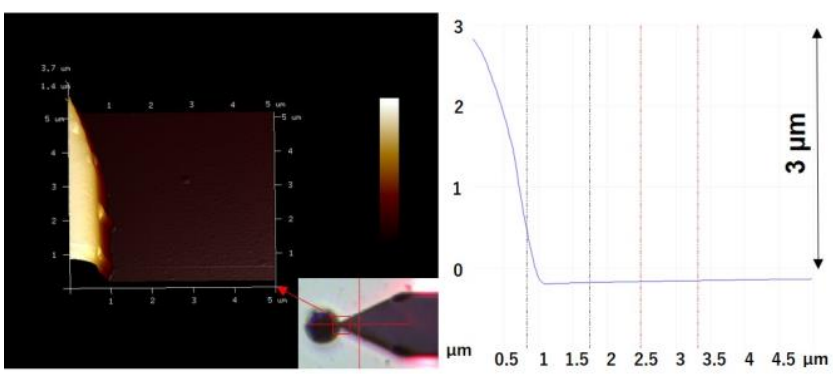

Fig. 7. Scanning topography around the bottom supporting ring for a fine position. The scanned topographical image of AFM and the measured topographical profile.

easy detachments of the helical microstructures by AFM cantilever without losing the mass thus we can expect minimal perturbation of propulsion performance (Fig. 2). An array of the helical microstructures with a minimum spacing between each structure was fabricated for their repeatable measurements and manipulation by AFM tip.

After the metallization step, the thickness of the deposited metal layers could be measured by an AFM scanning on an area over the interface between the masked (not deposited) area and the deposited area. The Figure 3 shows a scanned profile of the deposited metal layers. The thickness of the metallic layers was measured to be around $216 \mathrm{~nm}$ and this confirms the metallic layers were sufficiently deposited close to the aimed thickness. In addition, the surface roughness analyses of the deposited metallic layers could also be made by the scanning an area of 3 $\mu \mathrm{m}$ by $3 \mu \mathrm{m}$. Figure 4 shows the scanned image and the roughness $R_{a}$ and $R_{q}$ were $2.13 \mathrm{~nm}$ and $2.82 \mathrm{~nm}$ respectively which are around $1 \%$ of the layer thickness.

\section{AFM MiCROMANIPULATION PROCESS}

\section{A. Process Overview}

The fabricated microstructures and the target substrate are loaded onto the positioning stage of AFM. Since the fabricated microstructures are fixed on the substrate, several micromanipulation steps are required to prepare for the mass measurements. This includes a detachment of the helical structure out of the substrate and an attachment to AFM tip. Figure 5 summarizes the process overview to measure the mass of the fabricated microstructures. It starts from the engagement of an AFM tip to the substrate to make a coarse positioning. Then a microstructure is detached from the substrate and attached to the AFM tip. Once the mass measurement is done, the microstructure is redeposited onto the substrate either vertically or horizontally for their further analysis by SEM imaging. Furthermore, after the measurement, they can also be integrated to microfluidic chips for their microfluidic propulsion tests and manipulation experiments.

\section{B. Detachment of Microstructures from Substrate}

Figure 6 shows the optical microscope image of the AFM manipulation. The microstructure detachment out of the fabrication substrate is important but conventional micromanipulation approaches are not free from the addition or loss of mass mainly due to the less-controlled manipulation or the chemical adhesive. Therefore, here we propose a chemical-free and controlled detachment process. The specially designed 6 microneedle array support to maintain a 3D helical microstructure with minimum surface contact. The microneedle supports are conical shape with sufficient mechanical stability to maintain the helical microstructures during fabrication and transport processes. And it also helps to minimize the contact area which is the main source of potential mass addition or loss during the detachment process.

In addition, the micromanipulation process to detach the testing microstructure could further be improved in terms of the manipulation precision. An AFM tip is engaged near the supporting structure to avoid any potential damage. Then the tapping mode with scanning the interface area to the bottom 


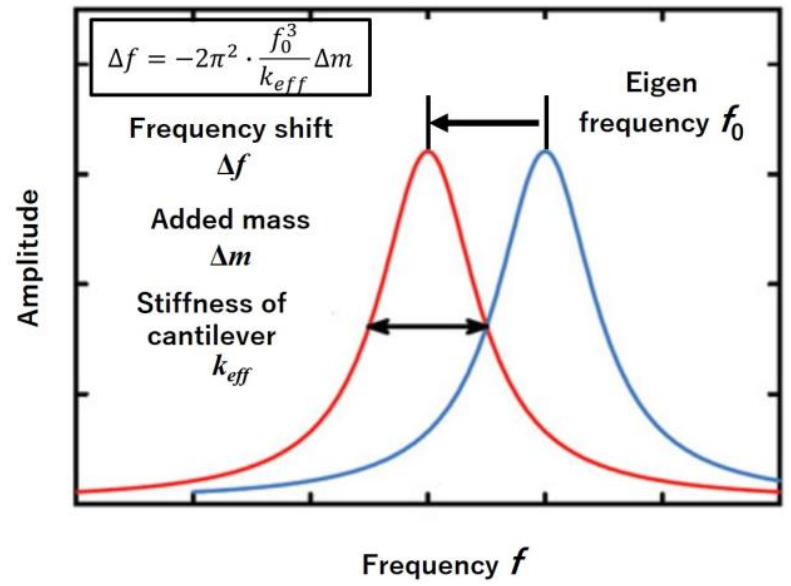

Fig. 8. Mass sensing by resonant frequency shift.

supporting ring structure. Based on the topographical image, the AFM tip can be precisely positioned on top of the bottom supporting ring. Skipping this positioning step, the AFM tip can apply excessive random force with vibration during the detachment step. This results in either damaging the helical microstructures or detaching the supporting structure together with the helical microstructure thus can lead to a wrong measurement. Figure 7 shows the scanned image and photograph of the AFM tip adjacent to the supporting ring. Based on the AFM`s topographical image, the AFM tip is moved on to the supporting ring without touching to the helical microstructure. Then the helical microstructure is disconnected from the supporting microneedles while moving the AFM tip further towards the center of the ring.

The disconnected helical microstructure is attracted electrostatically to the AFM tip. Once it is attached to the AFM tip, the AFM tip is moved vertically to $\mathrm{Z}$ axis to free the microstructure out of the substrate. The helical microstructure is attached with van der Waals force, it remains to be attached to the AFM tip. Once the microstructure is sufficiently far enough from the substrate, we perform the mass measurement at this step after shifting to the tapping mode. Once the measurement is done, we place the microstructure to the target substrate for further analysis by SEM or for the microfluidic chip integration. Figure 3 shows the sequence of the manipulation process.

\section{Attachment of AFM Probe and Microstructure}

It is also crucial to avoid any possible addition or loss of mass during the mass measurement process. One of the major sources of the addition of mass could be caused from the addition of chemical glue dispensed to fix the AFM tip and the testing microstructure. For this concern, van der Waals force is used for the attachment step without adding any chemicals. This allows the attachment/detachment process to be reversible. In addition, local electrostatic force induced from the charged AFM tip helps to maintain the attachment firmly during the handling and the measurement.

Completing the mass measurement, the tested microstructure should be released out of the AFM tip for the next measurements and also for the further integration to

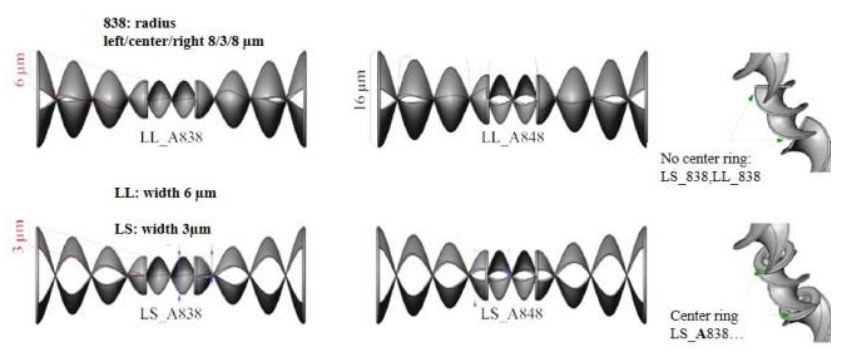

Fig. 9. Testing samples with geometrical variants.

TABLE II

COMPARISON WITH MEASURED AND CALCUlated MASS OF MicrohELIX

\begin{tabular}{|c|c|c|c|c|}
\hline Sample & $\begin{array}{c}\text { Frequency } \\
\Delta \mathrm{f}(\mathrm{Hz})\end{array}$ & $\begin{array}{c}\text { Sensitivity } \\
(\mathrm{Hz} / \mathrm{pg})\end{array}$ & $\begin{array}{c}\text { Measured } \\
\text { Mass (ng) }\end{array}$ & $\begin{array}{c}\text { Calculated } \\
\text { Mass (ng) }\end{array}$ \\
\hline LL_838 & 40750 & $25 \pm 1$ & 1.65 & 3.48 \\
\hline LL_A848 & 53618 & $25 \pm 1$ & 2.14 & 4.05 \\
\hline LS_A838 & 35339 & $25 \pm 1$ & 1.41 & 3.01 \\
\hline LL_A838 & 44492 & $24 \pm 1$ & 1.82 & 3.61 \\
\hline LS_838 & 32077 & $24 \pm 1$ & 1.33 & 2.88 \\
\hline LS_A848 & 39176 & $25 \pm 1$ & 1.58 & 3.31 \\
\hline
\end{tabular}

microfluidics. This can be made by placing the microstructure onto the substrate thanks to the fact that the microstructure`s contact surface area to the substrate is higher than the one to the AFM tip (Fig. 6). Moreover, an engagement of the AFM tip to a substate will apply minimal force sufficient to deflect the helical microstructures attached to the AFM tip. This allows to disconnect the microstructure's contact to the AFM tip. The remaining surface charge induced electrostatic force can also be reduced by discharge to the substrate when the AFM tip with the microstructure is in contact with substrate. This will help the microstructure to be released easily on the substrate. Furthermore, the touching force and speed of the AFM tip to substrate can be controlled through the GUI of AFM to find optimal releasing condition in the substrates with different surface energy.

\section{Mass MEASUREMENT AND ANALYSIS}

\section{A. Mass Measurement by Resonant Frequency Shift}

Mass measurement and further analysis of 3D helical microstructure is described in this section. Mass measurement

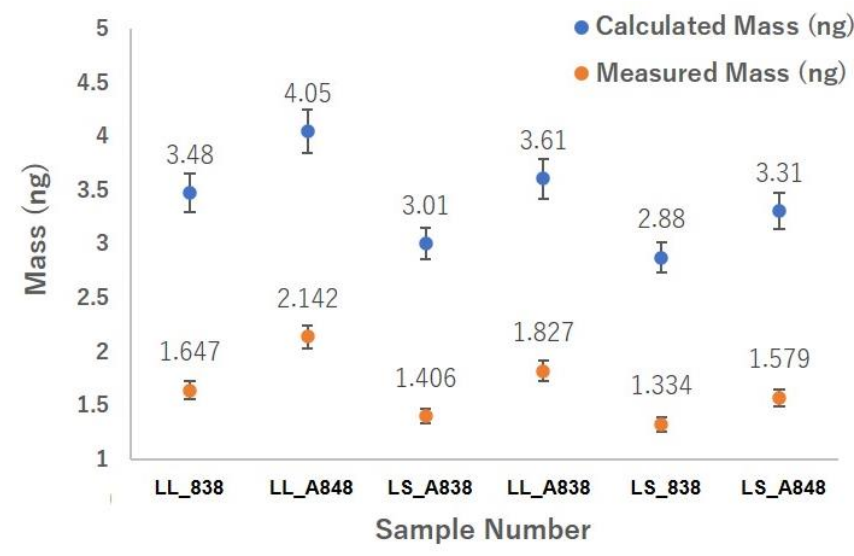

Fig. 10. Comparison with measured and calculated mass. 


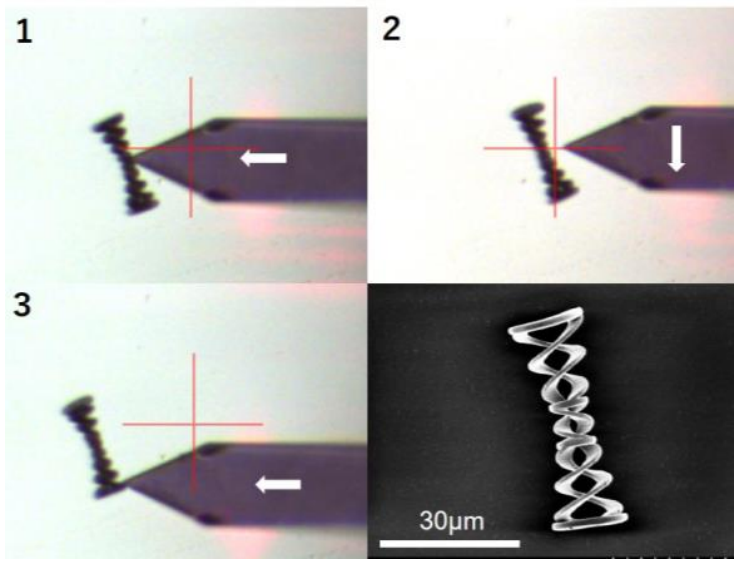

Fig. 11. Morphology and defect analysis by SEM imaging by rolling 3D helical microstructure after its mass measurement.

is performed by measuring the shift of the resonant frequency of the AFM tip with and without the helical microstructure. As explained in the section III, several micromanipulation steps are necessary to be ready for the mass measurement. For the mass measurement, an AFM tip should first affect a thermal calibration in the tapping mode of AFM to characterize the resonant frequency of the AFM tip. Once the target helical microstructure is attached to the AFM tip by contact mode AFM, we shift the AFM to tapping mode. Then another thermal calibration is performed to characterize the resonant frequency of the AFM cantilever with added mass of target sample. The addition of the microstructure's mass $\Delta m$ which is much smaller than the cantilever causes the shift in the resonant frequency $\Delta f$. With the constant stiffness $\left(k_{e f f}\right)$ of the cantilever, the resonant frequency shift $(\Delta f)$ is expressed by the first order approximation [22]:

$$
\Delta m=\frac{\Delta f \cdot k_{e f f}}{-2 \pi^{2} \cdot f^{3}}
$$

After each measurement, the AFM tip with the 3D helical microstructure moves to the target substrate for their microfluidics integration. If necessary, the measured samples can be measured again by the same measurement process (Fig. 6). This pick-measure-place can be repeatedly performed with same or different samples even in next days. At each time of the measurement, the resonant frequencies of AFM tip are thermal calibrated to assure the stable mass measurements for long-term measurements. This measurement method could be useful to calibrate the mass prior to their propulsion applications and also to re-calibrate the mass after being used in microfluidic applications.

Figure 9 shows the tested helical microstructure samples. The 6 different types of samples were prepared by the fabrication method described in the section II. The testing samples with different radius and width are shown in the Figure 9. We revealed the mass of each and the measurement sensitivity (Table II). The mass was ranging from $1.334 \mathrm{ng}$ to $2.142 \mathrm{ng}$ and the sensitivity was sufficiently high around 25 $\mathrm{Hz} / \mathrm{pg}$. Iterative measurements of identical testing microstructure with pick-and-place manipulations showed that

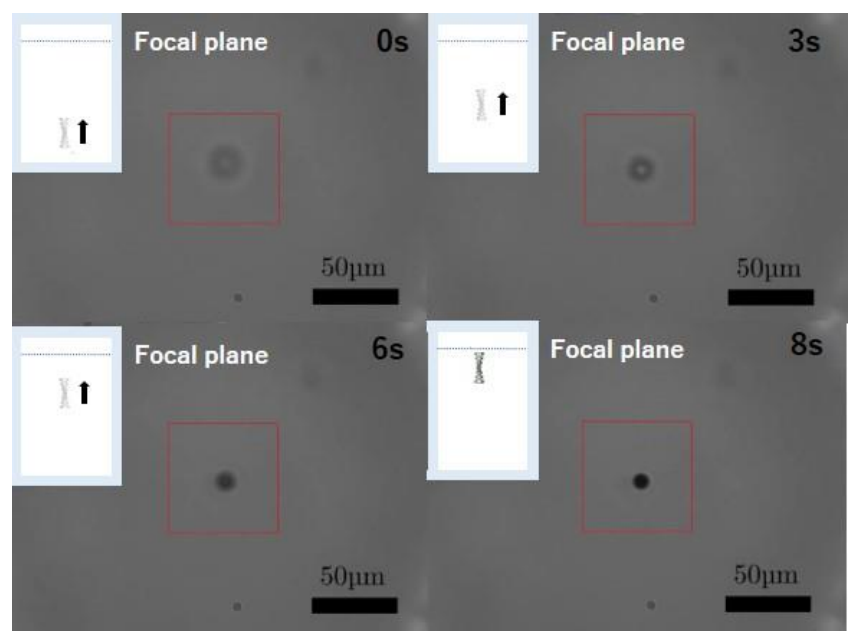

Fig. 12. Gravity compensation by swimming in vertical axis.

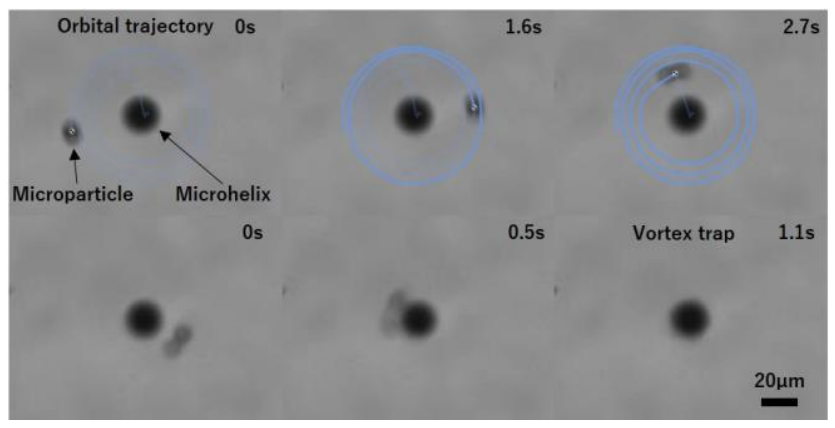

Fig. 13. Hydrodynamic manipulation of microparticle by vortical flow generated from rotating microhelix.

the deviation of the measured mass is within the sensitivity. This shows that each measurement did not add or lose a critical mass for the helical microstructures.

We compared the measured mass with the calculated mass estimated in our previous work [23]. In all the 6 models, the measured mass and the calculated one correspond well but with offset errors. We can consider that the calculated mass could contain the errors particularly in the volume estimations of the lithography structure and the deposited metal layers.

In addition to the mass measurement shown in this section and the surface topography analysis shown in the section II, further analysis on the defects or morphological deformations could also be performed by rolling the 3D helical microstructures (Fig. 11). We placed a testing structure laterally on the substrate and used AFM tip to push to roll the structures by contact mode AFM. The SEM imaging analysis revealed that the tested microstructure did not have any major deformations or damage from the whole process of manipulation and measurement. This step can validate qualitatively that the proposed process is minimally destructive thus promising for repeatable mass calibrations of $3 \mathrm{D}$ complex and composite microstructures.

\section{SWIMMING AND NON-CONTACT MiCROMANIPULATION}

The helical microstructures are promising for 3D non-contact micromanipulation tool inside microfluidic 


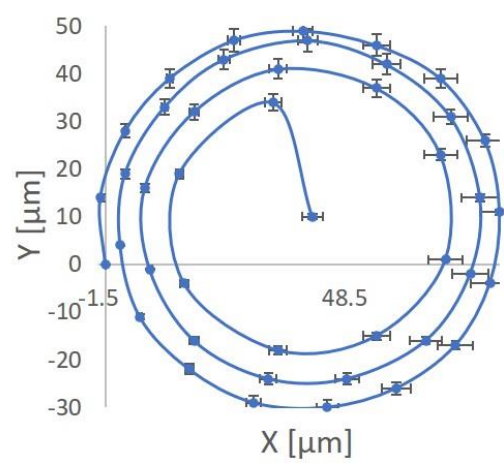

Fig. 14. Spiral trajectory of a microparticle by vortical flow from rotating helical microswimmer.

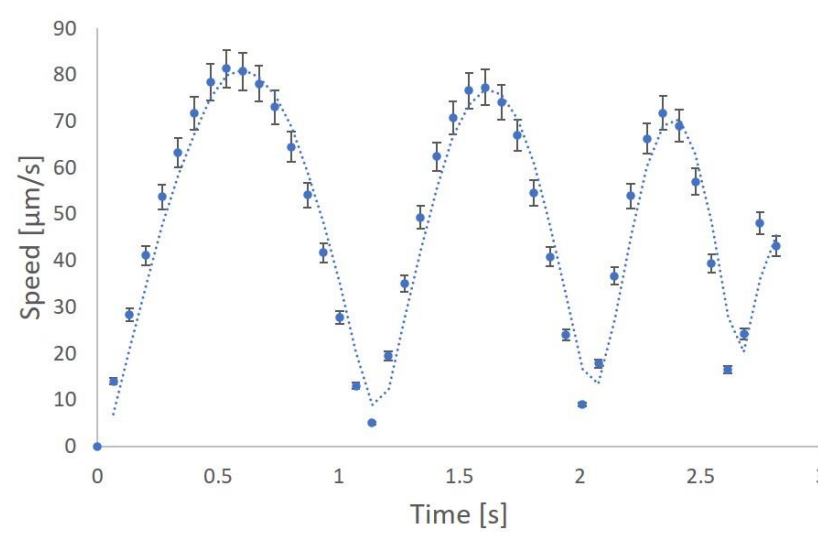

Fig. 15. Speed characterization of the moving microparticle by vortical flow from rotating helical microswimmer.

environments. Propelling the microswimmers with weak external magnetic field could have a great advantage particularly in the biological cell manipulation. Our previous works demonstrated 2D non-contact micromanipulation based on the vortex trap generated by rotating helical microswimmers [12]. It was recently extended to $3 \mathrm{D}$ non-contact manipulation by further elaborating and optimizing the design of the helical microswimmers [23]. Here we experimentally measured the mass of the 6 different modes of such 3D helical microswimmers and integrated each of them to microfluidics by AFM micromanipulation. To prove the integration process, we demonstrated the 3D propulsion by gravity compensation and the non-contact micromanipulation by the helical microswimmers integrated to microfluidics. It should be noted that they are the two major functionalities of the helical microswimmers for microfluidics applications.

Once the helical microswimmers are integrated to microfluidics, we injected water to fill the chip. We first demonstrated the gravity compensation of the helical microswimmers by swimming vertically in $\mathrm{Z}$ axis. All the tested models were able to swim against the gravity. Figure 12 shows the photos of the gravity compensation of one model (LS_838) of the helical microswimmers. The inner height of the fluidic chamber was $1 \mathrm{~mm}$ and the focal plane of the camera was set to $270 \mu \mathrm{m}$. The microswimmer was able to swim against the gravity from the rotating frequency of around $70 \mathrm{~Hz}$.
The propulsion speed was linearly proportional to the rotating frequency in the range of the rotating frequencies between 70 $\mathrm{Hz}$ and $200 \mathrm{~Hz}$. The propulsion speed of the model (LS_838) at a rotating frequency $(150 \mathrm{~Hz})$ in $\mathrm{Z}$-axis was measured around $33.8 \mu \mathrm{m} / \mathrm{s}$. It should be noted that the helical microswimmer was able to reach to the top surface of the fluidic chamber.

In addition to the swimming by gravity compensation, we also demonstrated the non-contact particle manipulation by vortex trapping (Fig. 13). Polystyrene microparticles with a diameter of $10 \mu \mathrm{m}$ were injected to the fluidic chamber together with helical microswimmer. The helical microswimmer was rotated around the Z-axis and attracted single microparticle by the vortex generated from the rotation. The particle started to move through a spiral trajectory and got closer to the rotating helical microswimmer at each cycle of rotation. The vortex trapping of the particle becomes stable when the particle gets to be synchronized to the rotation of the microswimmer. The spiral trajectory of the microparticle is plotted in the Figure 14. This trajectory was obtained from the tracked position of the microparticle from camera mounted on zoom optics. The distance from the microparticle to the center of rotating axis was characterized to be in the range between $25 \mu \mathrm{m}$ and $40 \mu \mathrm{m}$. This can visualize the vortex generated from the rotating helical microswimmer to help optimize the stability of vortex trapping. In addition, the speed of the microparticle`s motion in the spiral trajectory shows the cyclic behavior of the speed up to around $80 \mu \mathrm{m} / \mathrm{s}$ (Fig. 15). Such unstable vortex manipulation was observed over $50 \mathrm{~Hz}$ of rotating frequency of microswimmer. By such analysis, we can characterize and choose a right range of rotating frequency for a stable vortex trap manipulation.

With the demonstrations of characterizing the two major functionalities (gravity compensation and hydrodynamic manipulation) of helical microswimmers for microfluidics applications, we can consider that the proposed mass measurement and integration process to microfluidic chip by the proposed AFM micromanipulation is a valid manufacturing process.

\section{CONCLUSIONS}

We proposed a direct mass measurement and a microfluidic integration method by AFM micromanipulation for 3D complex shape composite microstructures. We tested the method with 3D helical microswimmers. A specially designed microneedle array supporting structure allowed the vertically fabricated 3D helical microswimmers and the uniform metallic layers depositions by sputtering. It also allowed the helical microstructures to be easily detached from fabrication substrate and to be attached to an AFM tip by AFM micromanipulation. The mass measurement was made by measuring the resonant frequency shift with a sensitivity around $15 \mathrm{~Hz} / \mathrm{pg}$. Since the proposed method is a purely physical manipulation without using chemical adhesive but by the van der Waals force adhesion, the measurement process can be reversible. The tested microstructures can be placed back to the fabrication substrate or other substrates to conserve for further measurements and also for further analyses by electron microscopy. Moreover, the measured 3D helical structures can 
be integrated to microfluidic chips by the controlled AFM micromanipulation for their quality characterizations of microfluidic propulsion and manipulation. The proposed AFM based direct mass measurement and the microfluidic integration method allowed a rapid, repeatable and less-destructive measurement and integration process. This can be an asset to manufacture the integrated microswimmers inside microfluidics thus to bring them closer to their biological or biomedical applications.

\section{ACKNOWLEDGMENT}

A part of this work is supported by Japan Society for the Promotion of Science (JSPS) through invitational fellowships for research in Japan and also by the JSPS Core-to-Core Program (A. Advanced Research Networks). The test microstructure design, 3D nanolithography and AFM measurements were supported by the French RENATECH network through its $\mathrm{C} 2 \mathrm{~N}$ technological facility. Metallic layer deposition by sputtering was supported by MEXT/VDEC Nanotechnology Platform in The University of Tokyo.

\section{REFERENCES}

[1] J. K. Gansel, M. Thiel, M. S. Rill, M. Decker, K. Bade, V. Saile, G. V. Freymann, S. Linden, and M. Wegener, "Gold helix photonic metamaterial as broadband circular polarizer" Science, vol. 325, no. 5947, pp. 1513-1515, Sep. 2009.

[2] K. Kamata, S. Suzuki, M. Ohtsuka, M. Nakagawa, T. Iyoda, and A. Yamada, "Fabrication of left-handed metal microcoil from spiral vessel of vascular plant" Adv. Mater., vol. 23, pp. 5509-5513, Oct. 2011.

[3] A. K. Raub and S. R. J. Bruek, "Large area 3D helical photonic crystals" $J$. Vac. Sci. Technol. B, vol. 29, no. 6, pp. 06ff02, Nov. 2011.

[4] D. J. Bell, L. Dong, B. J. Nelson, M. Golling, L. Zhang, and D. Grutzmacher, "Fabrication and Characterization of Three-Dimensional InGaAs/GaAs Nanosprings" Nano Lett., vol. 6, no. 4, pp. 725-729, Mar. 2006.

[5] G. Hwang, H. Hashimoto, D. J. Bell, L. Dong, B. J. Nelson, and S. Schon, "Piezoresistive InGaAs/GaAs Nanosprings with Metal Connectors" Nano Lett., vol. 9, no. 2, pp. 554-561, Jan. 2019.

[6] L. Dong, L. Zhang, B. E. Kratochvil, K. Shou, and B. J. Nelson, "Dual-chirality helical nanobelts: linear-to-rotary motion converters for three-dimensional microscopy" J. Microelectromech. Syst., vol. 18, no. 5, pp. 1047-1053, Oct. 2009.

[7] G. Hwang, R. Braive, L. Couraud, A. Cavanna, O. Abdelkarim, I. Robert-Philip, A. Beveratos, I. Sagnes, S. Haliyo, and S. Regnier, "Electro-osmotic propulsion of helical nanobelts swimmers" Intl. J. Rob. Res., vol. 30, no. 7, pp. 806-819, June 2011.

[8] K. Robbie and M. J. Brett, "Sculptured thin films and glancing angle deposition: Growth mechanics and applications" J. Vac. Sci. Technol. A, vol. 15, no. 3, pp. 1460-1465, 1997.

[9] S. Maruo, O. Nakamura, and S. Kawata, "Three-dimensional microfabrication with two-photon-absorbed photopolymerization" Opt. Lett., vol. 22, no. 2, pp. 132-134, 1997

[10] J. K. Gansel, M. Latzel, A. Frolich, J. Kaschke, M. Thiel, and M. Wegener, "Tapered gold-helix metamaterials as improved circular polarizers" Appl. Phys. Lett., vol. 100, pp. 101109, 2012.

[11] S. Tottori, L. Zhang, F. Qiu, K. K. Krawczyk, A. Franco-Obregon, and B. Nelson, "Micromachines: Magnetic Helical Micromachines: Fabrication, Controlled Swimming, and Cargo Transport" Adv. Mat., vol. 24, no. 6, pp 811-816, Feb. 2012.

[12] A. Paris, D. Decanini, and G. Hwang, "On-chip multimodal vortex trap micro-manipulator with multistage bi-helical micro-swimmer" Sens. Act.: A. Phys., vol. 276, pp. 118-124, June 2018.

[13] A. Barbot, D. Decanini, and G. Hwang, "Helical microrobot for force sensing inside microfluidic chip" Sens. Act.: A Phys., vol. 266, pp. 258-272, 2017.

[14] A. Barbot, D. Decanini, and G. Hwang, "Local Flow Sensing on Helical Microrobot for Semi-Automatic Motion Adaptation" Intl. J. Rob. Res., vol. 39, no. 4, pp. 476-489, 2020.
[15] X. Chen, J. Liu, M. Dong, L. Muller, G. Chatzipirpiridis, C. Hu, A. Terzopoulou, H. Torlakcik, X. Wang, F. Mushtaq, J. Puigmarti-Luis, Q. Shen, B. J. Nelson, S. Pane, "Magnetically driven piezoelectric soft microswimmers for neuron-like cell delivery and neuronal differentiation" Mater. Horiz, vol. 6, pp. 1512, 2019.

[16] H. Huang, F. E. Uslu, P. Katsamba, E. Lauga, M. S. Sakar, B. J. Nelson, "Adaptive locomotion of artificial microswimmers" Sci. Adv., vol. 5, no. 1, eaau1532, 2019.

[17] Y. Dong, L. Wang, J. Wang, S. Wang, Y. Wang, D. Jin, P. Chen, W. Du, L. Zhang, B. Liu, "Graphene-Based Helical Micromotors Constructed by "Microscale Liquid Rope-Coil Effect" with Microfluidics" ACS Nano, vol. 14, no. 12, pp. 16600-16613, 2020.

[18] K. Park, N. Kim, D. T. Morisette, N. R. Aluru, and R. Bashir, "Resonant MEMS Mass Sensors for Measurement of Microdroplet Evaporation" $J$. Microelectromech. Syst., vol. 21, no. 3, pp. 702-711, June 2012.

[19] P. Joshi, S. Kumar, V. K. Jain, J. Akhtar, and J. Singh, "Distributed MEMS Mass-Sensor Based on Piezoelectric Resonant Micro-Cantilevers" J. Microelectromech. Syst., vol. 28, no. 3, pp. 382-389, June 2019.

[20] J. C. Acosta, G. Hwang, J. Polesel-Maris, and S. Regnier, "A tuning fork based wide range mechanical characterization tool with nanorobotic manipulators inside a scanning electron microscope" Rev. Sci. Instrum., vol. 81, pp. 035116, Mar. 2011

[21] G. Hwang, C. David, A. Paris, D. Decanini, A. Mizushima, and Y. Mita, "A Rapid, Reliable and Less-destructive On-chip Mass Measurement for 3D Composite Material Testing Microstructures" 2020 IEEE 33rd Intl. Conf. Microelectron. Test Struct. (ICMTS), Edinburgh, United Kingdom, pp. 1-4, 2020.

[22] M. Mauro, R. Battaglia, G. Ferrini, R. Puglisi, D. Balduzzi, and A. Galli, "Single microparticles mass measurement using an AFM cantilever resonator" arXiv : vol. 1410, pp. 1953, Oct. 2014.

[23] A. Paris, D. Decanini, and G. Hwang, "Swimming force characterizations of multistaged bi-helical microswimmer and 3D vortex trap manipulation" Microelectron. Eng., vol. 235, pp. 111466, 2021. 